Neuer lang wirksamer Bronchodilatator mit einfacher Handhabung

\title{
Einmal tägliche Inhalation für bessere Compliance
}

- Der lang wirksame Beta-2-Agonist Indacaterol $\left(\right.$ Onbrez $^{\circledR}$, im Breezhaler $\left.{ }^{\circledR}\right)$ wirkt nach einer Inhalation rasch und rund um die Uhr und ist leicht zu handhaben.

Indacaterol vereine die Vorzüge von Formoterol und Salmeterol, weil es hydro- und lipophile Anteile hat, so Prof. Adrian Gillissen, Leipzig. „Deshalb setzt die Wirkung relativ schnell ein, hält aber 24 Stunden an. "Beides wurde überzeugend in Studien belegt. So erhöhten einmal täglich 150 bzw. $300 \mu \mathrm{g}$ Indacaterol schon fünf Minuten nach der Inhalation das Einsekundenvolumen FEV signifikant gegenüber Placebo: Das FEV mit Indacaterol lag 130 bzw. $140 \mathrm{ml}$ über dem der Kontrollgruppe ( $p<0,001$; Studie INTIME [1]).

Primärer Endpunkt in den meisten Untersuchungen war der Through-FEV ${ }_{1}$. Er lag in den Studien INTIME, INLIGHT-1 [2] und -2 [3] sowie INHANCE bzw. INVOLVE [4] mit
Indacaterol um 140 bis $180 \mathrm{ml}$ signifikant höher als mit Placebo. Auch der neue Beta2-Agonist war in diesem Parameter den Vergleichssubstanzen Tiotropium (18 $\mu \mathrm{g}$ qd, INTIME), Salmeterol (50 $\mu \mathrm{g}$ bid, INLIGHT-2) oder Formoterol ( $12 \mu \mathrm{g}$ bid, INVOLVE) zumindest nicht unterlegen, z. T. signifikant überlegen. Meist wurde der neue Wirkstoff in den in Deutschland zugelassenen Dosen von 150 oder $300 \mu \mathrm{g}$ qd eingesetzt, in INVOLVE bis zu $600 \mu \mathrm{ggd}$.

\section{Regelmäßige Anwendung wichtig}

„Für den Erfolg ist aber regelmäßige Anwendung wichtig“, so Prof. Heinrich Worth, Fürth. Die Therapieadhärenz für COPD-Medikamente sei selbst nach COPD-bedingter Hospitalisierung mit 33-61\% niedrig.,,Hier kommt ein weiterer Vorteil des neuen Medikaments zum Tragen“, so Gillissen: „Es muss nur einmal täglich inhaliert werden, und der zugehörige Inhalator ist leicht zu handhaben." Der Wirkstoff ist in einer transparenten Kapsel, die eingelegt und angestochen wird. Nach dem Atemzug kann der Patient kontrollieren, ob alles verbraucht wurde. Der niedrige Atemzugswiderstand mache den Breezhaler ${ }^{\circledR}$ geeignet für alle COPD-Patienten.

Gillissen lobte die gute Verträglichkeit von Indacaterol: „Sie ist der anderer lang wirksamer Beta-2-Mimetika ähnlich." An unerwünschten Effekten könne v.a. Husten kurz nach der Inhalation auftreten.

rdf

1. Vogelmeier $C$ et al. Chest 2009;136:4S-5S.

2. Feldman G et al. Am J Respir Crit Care Med 2009. 9:A4548.

3. Kornmann O et al. Chest 2009. 136:152S. 4. Dahl et al, ERS 2009

Symposium beim DGIM-Kongress, 12.4.2010 in Wiesbaden, Veranstalter: Novartis

\section{Pulmonalarterielle Hypertonie}

\section{Spezifische Therapie setzt meistens zu spät ein}

- Mit der Verfügbarkeit von Prostanoiden, Endothelinrezeptor-Antagonisten und Phosphodiesterase-(PDE-)5-Hemmern hat sich die pulmonalarterielle Hypertonie (PAH) von einer tödlichen zu einer kontrollierbaren chronischen Erkrankung gewandelt. Prognoseverbessernd wirkt ein Therapiebeginn im stabilen Stadium mit weitgehend erhaltener Rechtsherzfunktion. Wie Prof. Ardeschir Ghofrani erklärte, können Patienten mithilfe der $\mathrm{PAH}$-spezifischen Therapie heute auch langfristig stabilisiert werden. Angestrebt werden ein funktioneller Status NYHA $\leq$ II und eine 6Minuten-Gehstrecke $\geq 380$ Metern. Eine medikamentöse Option, die seit Kurzem zu diesem Zweck eingesetzt wird, ist der PDE5-Hemmer Tadalafil (Adcirca ${ }^{\circledR}$ ). Die Gabe der Substanz besserte in der PHIRST-1-Studie mit 16 Wochen Laufzeit die körperliche Belastbarkeit der Patienten vs. Placebo signifikant (Abb. 1). Mit Tadalafil zeigte sich zudem eine insgesamt bessere kardiopulmonale Hämodynamik und seltener eine klinische Verschlechterung. In der doppelblinden
Anschlussstudie PHIRST 2 wurde nach weiteren 52 Wochen demonstriert, dass der Effekt von Tadalafil auf die körperliche Belastbarkeit langfristig anhält.

Die Substanz wird in einer Dosierung von einmal $40 \mathrm{mg} / \mathrm{d}$ im Therapiealgorithmus der ERS/ESC bei Patienten in den Funktionsklassen II und III empfohlen.

Die Diagnose PAH wird aber heute in drei von vier Fällen erst in den Funktionsklassen

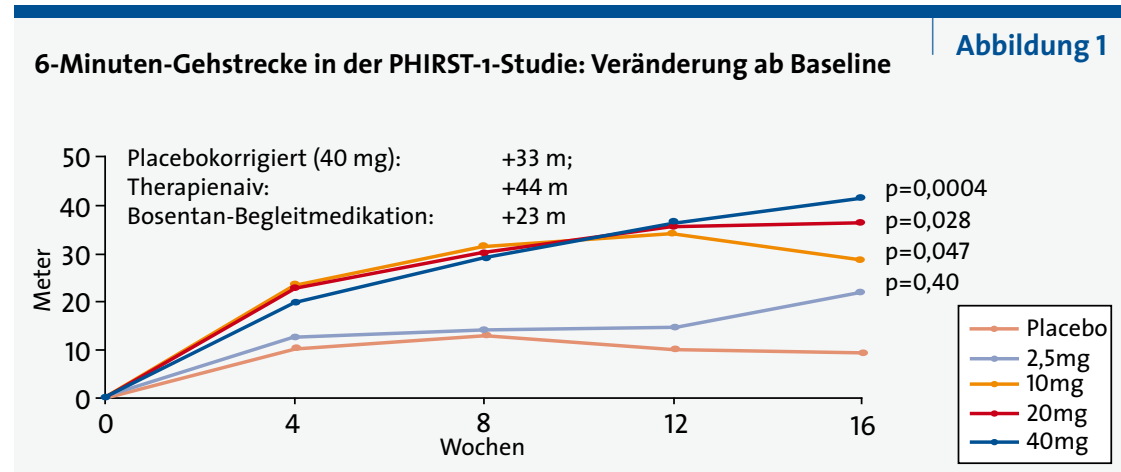

Die durchschnittlich verlängerte Gehstrecke bei therapienaiven Patienten lag mit Tadalafil 40 mg zu Studienende bei 44 Metern.
NYHA III und IV gestellt. Die erweiterten Therapieoptionen bei PAH könnten durch eine verbesserte Früherkennung noch viel besser ausgeschöpft werden. Gibt es echokardiografisch Hinweise auf Probleme im Lungenkreislauf, sollten sie in einem Spezialzentrum subtil abgeklärt werden. dn

Pressegespräch im Uniklinikum Gießen, 9.3.2010. Veranstalter: Lilly 